\title{
Asymmetric Allylboration of Aldehydes and Ketones using 3,3'-Disubstitutedbinaphthol-modified Boronates
}

\author{
T. Robert Wu, Lixin Shen, and J. Michael Chong* \\ Guelph-Waterloo Centre for Graduate Work in Chemistry and Biochemistry, $(G W C)^{2}$ \\ Department of Chemistry, University of Waterloo, Waterloo, Ontario, Canada \\ N2L $3 G 1$ \\ Supporting Information
}

\section{Table of Contents}

General Experimental...................................................... 2

Preparation of 2,2'-bis(methoxymethoxy)-1,1'-binaphthyl (2).......................S3

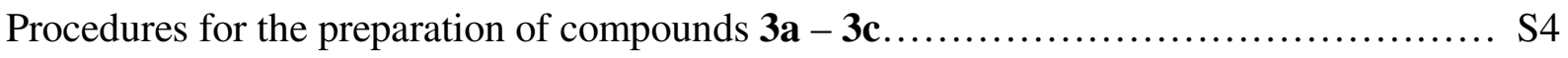

Procedures for the preparation of compounds $\mathbf{3 d}-\mathbf{3 i} \ldots \ldots \ldots \ldots \ldots \ldots \ldots \ldots \ldots \ldots \ldots \ldots \ldots \ldots \ldots \ldots$

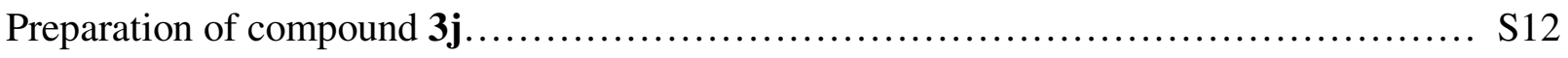

Procedures for the preparation of compounds $\mathbf{4 a}-\mathbf{4 j} \ldots \ldots \ldots \ldots \ldots \ldots \ldots \ldots \ldots \ldots \ldots \ldots \ldots \ldots$

General procedure for allylation of aldehydes and ketones....................... S20

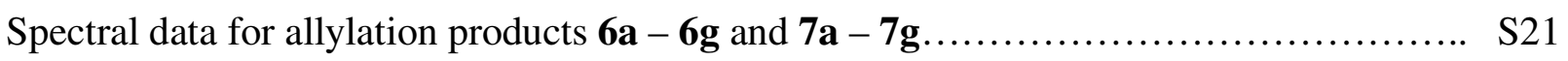

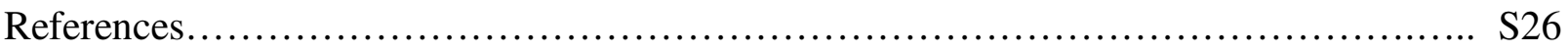




\section{General Experimental}

IR spectra were recorded on a Bomem MB-100 infrared spectrophotometer. ${ }^{1} \mathrm{H}(250$ and $300 \mathrm{MHz}),{ }^{13} \mathrm{C}(63$ and $75 \mathrm{MHz})$, and ${ }^{19} \mathrm{~F}$ (188 and $\left.182 \mathrm{MHz}\right) \mathrm{NMR}$ spectra were recorded in $\mathrm{CDCl}_{3}$ on Bruker AM250 or AVANCE 300 spectrometers unless otherwise noted. Chemical shifts are given in parts per million (ppm) downfield from tetramethylsilane (TMS). The ${ }^{1} \mathrm{H}$ NMR samples were run in deuteriochloroform containing ca. $0.01 \%$ TMS as an internal standard $(\delta=0.0)$. Spectra are tabulated in the order: chemical shift, assignment, numbers of protons, multiplicity, coupling constant. The spectral reference for ${ }^{13} \mathrm{C}$ NMR spectra was $\mathrm{CDCl}_{3}(\delta=77.0)$. The spectral reference for ${ }^{19} \mathrm{~F}$ NMR spectra was $\mathrm{CF}_{3} \mathrm{COOH}\left(\delta=76.53\right.$ upfield of $\left.\mathrm{CFCl}_{3}\right)$. Elemental analyses were conducted by M-H-W Laboratories, Phoenix, Arizona. Mass spectra were recorded on a GC-MS (Hewlett Parkard G 1800A GCD System) or a Kratos MA890 mass spectrometer using electron impact (EI, $70 \mathrm{ev)} \mathrm{ionization} \mathrm{if} \mathrm{the} \mathrm{mass} \mathrm{was} \mathrm{over} \mathrm{430.} \mathrm{Optical} \mathrm{rotations} \mathrm{were}$ recorded in cells with $10 \mathrm{~cm}$ path length on a Perkin-Elmer 241 digital polarimeter.

All reactions involving air or moisture sensitive reagents were performed under an argon or nitrogen atmosphere on the bench using standard Schlenk techniques. Solvents were dried prior to use. Tetrahydrofuran (THF) and diethyl ether $\left(\mathrm{Et}_{2} \mathrm{O}\right)$ were dried by distillation from sodium wire. Dichloromethane $\left(\mathrm{CH}_{2} \mathrm{Cl}_{2}\right)$, DMF, HMPA and $n$-pentane were dried by distillation from calcium hydride. Chloromethyl methyl ether was prepared according to the literature. ${ }^{1} \mathrm{BrCl}_{2} \mathrm{CCCl}_{2} \mathrm{Br}$ was prepared in $85 \%$ yield from the reaction of dichloroethene and bromine in dichloromethane under reflux condition for 3 days, followed by the removal of organic solvent. Triallylborane was prepared as reported by Brown. ${ }^{2}$ The triallylborane thus prepared was distilled prior to use so was metal-free. BINOL (1) was prepared following a literature procedure ${ }^{3}$ and was resolved using the Merck method. ${ }^{4}$ Methyl fluorosulfonyldifluoroacetate was purchased from SynQuest Laboratories, 
Alachua, FL and used without further purification. Unless otherwise noted, other chemicals were purchased from Aldrich Chemical Company.

\section{$( \pm)$ - and $(R)-2,2 '-B i s(m e t h o x y m e t h o x y)-1,1 '-B i n a p h t h y l(2)$.}

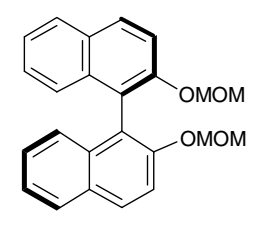

The preparation followed literature procedures ${ }^{5}$ with some modifications.

$\mathrm{NaH}(2.92 \mathrm{~g}, 60 \%$ in oil, $73.0 \mathrm{mmol})$ was mixed in dry THF $(150 \mathrm{~mL})$ in a $500 \mathrm{~mL}$ round bottom flask at $0{ }^{\circ} \mathrm{C}$ under an argon atmosphere. To the mixture with stirring, was added a solution of ( \pm )-2,2'-dihydroxy- 1,1'-binaphthyl (1) $(9.50 \mathrm{~g}, 33.2 \mathrm{mmol})$ in THF (50 mL) in a dropping funnel. After the addition, the mixture was stirred at $0{ }^{\circ} \mathrm{C}$ for $1 \mathrm{~h}$, then allowed to warm up to room temperature for $15 \mathrm{~min}$. After the mixture was re-cooled to $0{ }^{\circ} \mathrm{C}$, chloromethyl methyl ether $(5.54$ $\mathrm{mL}, 73.0 \mathrm{mmol}$ ) was slowly added from the dropping funnel. After the addition, the reaction mixture was warmed to room temperature and stirred for $4.5 \mathrm{~h}$. Saturated aqueous $\mathrm{NH}_{4} \mathrm{Cl}(50 \mathrm{~mL})$ was added to the flask, then the solvent was removed in vacuo. The residue was extracted with $\mathrm{CH}_{2} \mathrm{Cl}_{2}(50 \mathrm{~mL} \times 3)$. The organic layers were combined, washed with brine $(50 \mathrm{~mL})$, dried over $\mathrm{Na}_{2} \mathrm{SO}_{4}$, and concentrated. Crude product was purified by column chromatography (EtOAc/hexane: 1/10) and crystallized from $\mathrm{CH}_{2} \mathrm{Cl}_{2}$ /hexane to give a white crystalline product (士)-2 in quantitative yield. $\operatorname{mp~89-92~}{ }^{\circ} \mathrm{C}\left(\right.$ lit $\left.^{5} \mathrm{mp} 88-91{ }^{\circ} \mathrm{C}\right)$; IR (KBr): v (max) 1236, 1144, 1028 $\mathrm{cm}^{-1} ;{ }^{1} \mathrm{H} \mathrm{NMR}\left(\mathrm{CDCl}_{3}\right): \delta$ 7.88-8.14 (ArH, $\left.2 \mathrm{H}, \mathrm{m}\right)$, 7.76-7.84 (ArH, $\left.2 \mathrm{H}, \mathrm{m}\right)$, 7.10-7.62 (ArH, $8 \mathrm{H}$, m), 4.97, $5.08\left(\mathrm{OCH}_{2} \mathrm{O}, 4 \mathrm{H}, \mathrm{d}, \mathrm{AB}, \mathrm{J}_{\mathrm{AB}}=8.5 \mathrm{~Hz}\right), 3.10\left(\mathrm{OCH}_{3}, 6 \mathrm{H}, \mathrm{s}\right) ;{ }^{13} \mathrm{C} \mathrm{NMR}\left(\mathrm{CDCl}_{3}\right): \delta 152.6$, 130.0, 129.9, 129.3 127.8, 126.3, 125.5, 124.0, 121.3, 117.3, 95.2, 55.7; MS m/e (relative intensity): 
374 ( $\left.\mathrm{M}^{+}, 100\right), 298$ (90), 270 (71), 239 (23); Anal. Calcd for $\mathrm{C}_{24} \mathrm{H}_{22} \mathrm{O}_{4}$ : C, 76.99; H, 5.92. Found:

C, 77.02; H, 5.94. (R)-2,2'-Dihydroxy-1,1'-binaphthyl was used to afford (R)-2 in quantitative yield. mp $98-100{ }^{\circ} \mathrm{C}$. The sample showed $[\alpha]^{25}{ }_{589}+95.0^{\circ}(\mathrm{c}=1.0$, THF).

\section{General Procedure A: Preparation of 3,3'-Disubsituted-2,2'-Bis(methoxymethoxy) -1,1'-Binaphthyls 3a-3c.}

The preparation of 3,3'-disubstituted binaphthyl compounds followed literature procedures ${ }^{5}$ with some modifications.

(士)- or (R)-2,2'-Bis(methoxymethoxy)-1,1'-binaphthyl (2) (1 equiv) was dissolved in dry $\mathrm{Et}_{2} \mathrm{O}(17 \mathrm{~mL} / 1 \mathrm{mmol}$ of 2$)$ in a round bottom flask under an argon atmosphere. To the mixture with stirring, was added $n \mathrm{BuLi}$ ( 3 equiv) at room temperature by syringe injection. After the reaction mixture was stirred for $3 \mathrm{~h}$, THF ( $11 \mathrm{~mL} / 1 \mathrm{mmol}$ of 2 ) was injected to the flask and then the mixture was stirred for $1 \mathrm{~h}$. After the flask was cooled in an ice water bath for $5 \mathrm{~min}$, the appropriate electrophile (3 equiv) was quickly added in one portion. The reaction mixture was stirred for $15 \mathrm{~min}$, quenched with saturated aqueous $\mathrm{NH}_{4} \mathrm{Cl}$ and water. The two phases were separated. The aqueous layer was extracted with $\mathrm{Et}_{2} \mathrm{O}$ twice. All organic solutions were combined, washed with brine, dried over $\mathrm{Na}_{2} \mathrm{SO}_{4}$ and concentrated. Subsequent work-up gave the product.

( \pm )- and (R)-3,3'-Dibromo-2,2'-Bis(methoxymethoxy-1,1'-binaphthyl (3a).

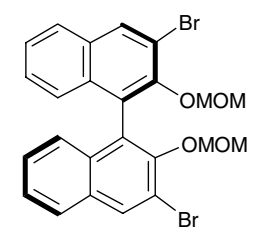

(士)-2,2'-Bis(methoxymethoxy)-1,1'-binaphthyl (2) $(8.80 \mathrm{~g}, 23.5 \mathrm{mmol})$ was treated with 
$n \mathrm{BuLi}(44.10 \mathrm{~mL}$ of a $1.6 \mathrm{M}$ solution in hexane, $70.5 \mathrm{mmol})$ at room temperature and resulting mixture was quenched with dibromotetrachloroethane $(23.0 \mathrm{~g}, 70.5 \mathrm{mmol})$. Crude product was purified by column chromatography (EtOAc/hexane: 1/10) and crystallized from $\mathrm{Et}_{2} \mathrm{O} / \mathrm{hexane}$ to give a white crystalline product $( \pm)-3 \mathbf{a}(10.60 \mathrm{~g})$ in $85 \%$ yield. $\mathrm{mp} 125-126^{\circ} \mathrm{C}\left(\mathrm{lit}^{5} \mathrm{mp} 124-126^{\circ} \mathrm{C}\right)$; IR (KBr): v (max) 1235, 1157, $1026 \mathrm{~cm}^{-1} ;{ }^{1} \mathrm{H}$ NMR $\left(\mathrm{CDCl}_{3}\right): \delta 8.25-8.30(\mathrm{ArH}, 2 \mathrm{H}, \mathrm{m}), 7.78-7.82$ (ArH, $2 \mathrm{H}, \mathrm{m}), 7.18-7.48(\mathrm{ArH}, 6 \mathrm{H}, \mathrm{m}), 4.81,4.82\left(\mathrm{OCH}_{2} \mathrm{O}, 4 \mathrm{H}, \mathrm{d}, \mathrm{AB}, \mathrm{J}_{\mathrm{AB}}=6.0 \mathrm{~Hz}\right), 2.56\left(\mathrm{OCH}_{3}\right.$, $6 \mathrm{H}, \mathrm{s}) ;{ }^{13} \mathrm{C} \mathrm{NMR}\left(\mathrm{CDCl}_{3}\right): \delta 153.2,141.0,133.6,131.8,131.6,127.2,126.7,126.6,126.2,125.6$, 99.8, 92.8, 56.6; MS m/e (relative intensity): $535\left(\mathrm{M}^{+}+3,20\right), 534\left(\mathrm{M}^{+}+2,18\right), 533\left(\mathrm{M}^{+}+1,4\right), 532$ $\left(\mathrm{M}^{+}, 9\right), 457$ (24), 455 (47), 453 (28), 376 (20), 268 (23), 45 (100); Anal. Calcd for $\mathrm{C}_{24} \mathrm{H}_{20} \mathrm{Br}_{2} \mathrm{O}_{4}$ : C, 54.16; H, 3.79. Found: C, 54.10; H, 3.87. (R)-2,2'-Bis(methoxymethoxy)-1,1'-binaphthyl was used to afford $(R)-3 \mathbf{a}$ in $84 \%$ yield. mp $123-124{ }^{\circ} \mathrm{C}$. The sample showed $[\alpha]^{25}{ }_{589}+28.3(\mathrm{c}=1.0$, THF).

\section{(士)- and $(R)-3,3$ '-Diiodo-2,2'-Bis(methoxymethoxy-1,1'-binaphthyl (3b).}

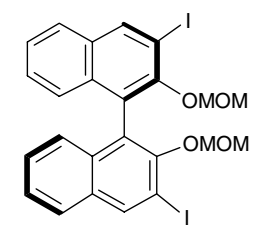

(士)-2,2'-Bis(methoxymethoxy)-1,1'-binaphthyl (2) (6.50 g, $17.36 \mathrm{mmol})$ was treated with $n \operatorname{BuLi}(33.0 \mathrm{~mL}$ of a $1.6 \mathrm{M}$ solution in hexane, $52.80 \mathrm{mmol})$ at room temperature and resulting mixture was quenched with iodine $(13.20 \mathrm{~g}, 52.0 \mathrm{mmol})$. Crude product was purified by column chromatography (EtOAc/hexane: 1/10) and crystallized from $\mathrm{CH}_{2} \mathrm{Cl}_{2} /$ hexane to give a white crystalline product $(3 \mathrm{~b})(9.60 \mathrm{~g})$ in $88 \%$ yield. $\mathrm{mp} 129-131{ }^{\circ} \mathrm{C}\left(\mathrm{lit}^{5} \mathrm{mp} 127-128{ }^{\circ} \mathrm{C}\right)$; $\mathrm{IR}(\mathrm{KBr}): v$ (max) 1232, 1156, $1026 \mathrm{~cm}^{-1} ;{ }^{1} \mathrm{H}$ NMR $\left(\mathrm{CDCl}_{3}\right): \delta$ 8.50-8.62 (ArH, $\left.2 \mathrm{H}, \mathrm{m}\right), 7.70-7.85(\mathrm{ArH}, 2 \mathrm{H}$, m), 7.10-7.54 (ArH, $6 \mathrm{H}, \mathrm{m}), 4.81,4.69\left(\mathrm{OCH}_{2} \mathrm{O}, 4 \mathrm{H}, \mathrm{d}, \mathrm{AB}, \mathrm{J}_{\mathrm{AB}}=5.7 \mathrm{~Hz}\right), 2.59\left(\mathrm{OCH}_{3}, 6 \mathrm{H}, \mathrm{s}\right)$; 
${ }^{13} \mathrm{C}$ NMR $\left(\mathrm{CDCl}_{3}\right): \delta 152.2,140.0,133.8,132.8,132.2,127.1,126.7,126.5,126.2,125.8,99.4$, 92.5, 56.5; MS m/e (relative intensity): 626 (M+3), 550 (20), 549 (100), 422 (60), 268 (45), 239 (46), 237 (28), 226 (37), 224 (45); Anal. Calcd for $\mathrm{C}_{24} \mathrm{H}_{20} \mathrm{I}_{2} \mathrm{O}_{4}$ : C, 46.03; H, 3.22. Found: C, 46.05; H, 3.20. (R)-2,2'-Bis(methoxymethoxy)-1,1'-binaphthyl was used to afford (R)-3b. mp $45-47{ }^{\circ} \mathrm{C}$. The sample showed $[\alpha]^{25}{ }_{589}-5.3(\mathrm{c}=1.0$, THF).

\section{(R)-3,3'-Dimethyl-2,2'-Bis(methoxymethoxy)-1,1'-Binaphthyl (3c).}

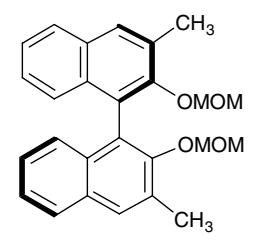

(R)-2,2'-Bis(methoxymethoxy)-1,1'-binaphthyl $[(R)-2](2.0 \mathrm{~g}, 5.34 \mathrm{mmol})$ was treated with $n$-BuLi $(10.0 \mathrm{~mL}$ of a $1.6 \mathrm{M}$ solution in hexane, $16.0 \mathrm{mmol})$ at room temperature and resulting mixture was quenched with iodomethane $(1.0 \mathrm{~mL}, 16.0 \mathrm{mmol})$. Crude product was purified by column chromatography (EtOAc/hexane: 1/10) and crystallized from $\mathrm{CH}_{2} \mathrm{Cl}_{2} /$ hexane to give a white crystalline product $(R)-3 \mathbf{c}(2.1 \mathrm{~g})$ in $96 \%$ yield. mp $91-92{ }^{\circ} \mathrm{C}\left(\right.$ lit $\left.^{5}(\mathrm{~S})-\mathbf{3 c} \mathrm{mp} 90.5-91.5{ }^{\circ} \mathrm{C}\right)$; IR (KBr): $v(\max ) 1238,1155,1035 \mathrm{~cm}^{-1} ;{ }^{1} \mathrm{H}$ NMR $\left(\mathrm{CDCl}_{3}\right): \delta$ 7.72-7.80 (ArH, $\left.4 \mathrm{H}, \mathrm{m}\right), 7.26-7.38$ $(\mathrm{ArH}, 2 \mathrm{H}, \mathrm{m}), 7.14-7.20(\mathrm{ArH}, 4 \mathrm{H}, \mathrm{m}), 4.46,4.58\left(\mathrm{OCH}_{2} \mathrm{O}, 4 \mathrm{H}, \mathrm{d}, \mathrm{AB}, \mathrm{J}_{\mathrm{AB}}=5.8 \mathrm{~Hz}\right), 2.83\left(\mathrm{OCH}_{3}\right.$, $6 \mathrm{H}, \mathrm{s}), 2.57\left(\mathrm{ArCH}_{3}, 6 \mathrm{H}, \mathrm{s}\right) ;{ }^{13} \mathrm{C} \mathrm{NMR}\left(\mathrm{CDCl}_{3}\right): \delta 153.1,133.0,131.6,131.0,129.6,127.1,126.1$, 125.5, 125.4, 124.7, 98.5, 56.4, 17.8; MS m/e (relative intensity): 402 ( $\left.\mathrm{M}^{+}, 30\right), 327$ (29), 326 (100), 309 (26), 298 (36), 297 (50), 296 (45), 283 (29), 252 (20), 239 (30), 119 (27), 111 (25); Anal. Calcd

for $\mathrm{C}_{26} \mathrm{H}_{26} \mathrm{O}_{4}: \mathrm{C}, 77.59 ; \mathrm{H}, 6.51$. Found: $\mathrm{C}, 77.55 ; \mathrm{H}, 6.47$. The sample showed $[\alpha]^{25}{ }_{589}-86.6(\mathrm{c}=$ $1.0, \mathrm{THF})$. 


\section{General Procedure B: Preparation of 3,3'-Diaryl-2,2'-Bis(methoxymethoxy)-1,1'-Binaphthyls}

\section{3d-3i.}

The preparation of 3,3'-disubstituted binaphthyl compounds followed literature procedures ${ }^{5}$ with some modifications.

(R)-3,3'-Dibromo-2,2'-Bis(methoxymethoxy)-1,1'-binaphthyl (3a) (1 equiv) and $\mathrm{Pd}\left(\mathrm{PPh}_{3}\right)_{4}$ (10 mol\%) were mixed in DME $(6.7 \mathrm{~mL} / 1 \mathrm{mmol}$ of $3 \mathbf{a})$ in a round bottom flask at room temperature under an argon atmosphere. To the mixture, with stirring, were added arylboronic acid (3.5 equiv) and $2 \mathrm{M}$ aqueous $\mathrm{Na}_{2} \mathrm{CO}_{3}$ solution (5.2 equiv). The resulting mixture was stirred and heated to reflux for $10 \mathrm{~h}$, cooled to room temperature, and passed through a pad of Celite. The organic solution was evaporated to give a residue. The residue was dissolved in $\mathrm{CH}_{2} \mathrm{Cl}_{2}$, washed with saturated aqueous $\mathrm{NH}_{4} \mathrm{Cl}$, water, brine, dried over $\mathrm{Na}_{2} \mathrm{SO}_{4}$, and concentrated to give a crude product. Subsequent purification gave the product.

(士)- and (R)-3,3'-Diphenyl-2,2'-Bis(methoxymethoxy)-1,1'-binaphthyl (3d).

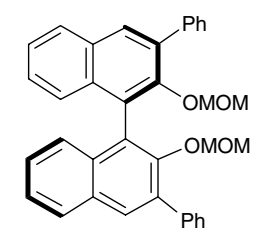

Following General Procedure B, ( \pm )-3,3'-Dibromo-2,2'-Bis(methoxymethoxy-1,1'binaphthyl (3a) (8.0 g, $15.0 \mathrm{mmol})$ was treated with $\mathrm{Pd}\left(\mathrm{PPh}_{3}\right)_{4}(1.73 \mathrm{~g}, 1.5 \mathrm{mmol})$, phenylboronic acid $(6.40 \mathrm{~g}, 52.6 \mathrm{mmol})$ and $2 \mathrm{M}$ aqueous $\mathrm{Na}_{2} \mathrm{CO}_{3}$ solution $(39 \mathrm{~mL})$ in DME $(100 \mathrm{~mL})$ in a $500 \mathrm{~mL}$ round bottom flask. Purification was carried by column chromatography (EtOAc/hexane: 1/10) to give a foamy product $( \pm)-3 \mathbf{d}(6.7 \mathrm{~g})$ in $85 \%$ yield. The product was directly used for deprotection of MOM group with only ${ }^{1} \mathrm{H}$ NMR and ${ }^{13} \mathrm{C}$ NMR being examined. ${ }^{1} \mathrm{H}$ NMR $\left(\mathrm{CDCl}_{3}\right): \delta 7.65-8.05$ 
$(\mathrm{ArH}, 8 \mathrm{H}, \mathrm{m}), 7.22-7.55(\mathrm{ArH}, 12 \mathrm{H}, \mathrm{m}), 4.40,4.37\left(\mathrm{OCH}_{2} \mathrm{O}, 4 \mathrm{H}, \mathrm{d}, \mathrm{AB}, \mathrm{J}_{\mathrm{AB}}=5.8 \mathrm{~Hz}\right), 2.34$ $\left(\mathrm{OCH}_{3}, 6 \mathrm{H}, \mathrm{s}\right) ;{ }^{13} \mathrm{C} \mathrm{NMR}\left(\mathrm{CDCl}_{3}\right): \delta 139.0,135.5,133.6,130.9,130.6,129.6,128.3,127.9,127.3$, $\begin{array}{llllll}126.5, & 126.4, & 126.3, & 125.2, & 98.5, & 55.8 .\end{array}$ (R)-3,3'-Dibromo-2,2'-Bis(methoxymethoxy)-1,1'-binaphthyl (3a) was used to afford $(R)$-3d.

\section{( \pm )- and $(R)-3,3$ '-Bis(4-methoxyphenyl)-2,2'-Bis(methoxymethoxy)-1,1'-binaphthyl (3e).}

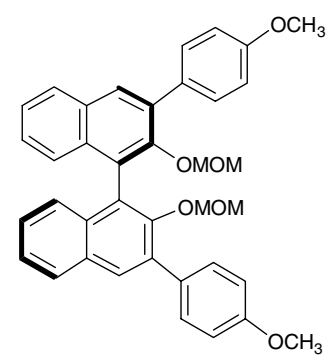

Following General Procedure B, ( \pm )-3,3'-Dibromo-2,2'-Bis(methoxymethoxy-1,1'binaphthyl (3a) $(1.0 \mathrm{~g}, 1.88 \mathrm{mmol})$ was treated with $\mathrm{Pd}\left(\mathrm{PPh}_{3}\right)_{4}(0.22 \mathrm{~g}, 0.188 \mathrm{mmol})$, 4-methoxyphenylboronic acid $(1.0 \mathrm{~g}, 6.58 \mathrm{mmol})$ and $2 \mathrm{M}$ aqueous $\mathrm{Na}_{2} \mathrm{CO}_{3}$ solution $(4.9 \mathrm{~mL})$ in DME $(20 \mathrm{~mL})$ in a $50 \mathrm{~mL}$ round bottom flask. Purification was carried by column chromatography (EtOAc/hexane: 1/10) and crystallization from $\mathrm{CH}_{2} \mathrm{Cl}_{2} /$ hexane to give colorless crystals (3e) $(1.53$ g) in $92 \%$ yield. mp $75-76{ }^{\circ} \mathrm{C}$; IR $(\mathrm{KBr}): v(\max ) 1608,1513,1247 \mathrm{~cm}^{-1} ;{ }^{1} \mathrm{H}$ NMR $\left(\mathrm{CDCl}_{3}\right): \delta$ 7.82-7.96 (ArH, 4 H, m), 7.63-7.78 (ArH, 4 H, m), 7.18-7.47 (ArH, 6 H, m), 6.90-7.08 (ArH, 4 H, $\mathrm{m}), 4.42,4.37\left(\mathrm{OCH}_{2} \mathrm{O}, 4 \mathrm{H}, \mathrm{d}, \mathrm{AB}, \mathrm{J}_{\mathrm{AB}}=5.7 \mathrm{~Hz}\right), 3.87\left(\mathrm{OCH}_{3}, 6 \mathrm{H}, \mathrm{s}\right), 2.35\left(\mathrm{OCH}_{3}, 6 \mathrm{H}, \mathrm{s}\right) ;{ }^{13} \mathrm{C}$ $\operatorname{NMR}\left(\mathrm{CDCl}_{3}\right): \delta$ 159.0, 151.3, 135.0, 133.4, 131.4, 130.9, 130.7, 130.2, 127.7, 126.6, 126.4, 126.1, 125.1, 113.8, 98.3, 55.9, 55.3; Anal. Calcd for $\mathrm{C}_{38} \mathrm{H}_{34} \mathrm{O}_{6}:$ C, 77.80; H, 5.84. Found: C, 77.68; H, 5.78. (R)-3,3'-Dibromo-2,2'-bis(methoxymethoxy)-1,1'-binaphthyl was used to afford (R)-yy8. mp $68-70{ }^{\circ} \mathrm{C}$. The sample showed $[\alpha]^{25}{ }_{578}-109.6(\mathrm{c}=1.0, \mathrm{THF})$. 
( \pm )- and (R)-3,3'-Bis(3,5-dimethylphenyl)-2,2'-Bis(methoxymethoxy)-1,1'-binaphthyl (3f).

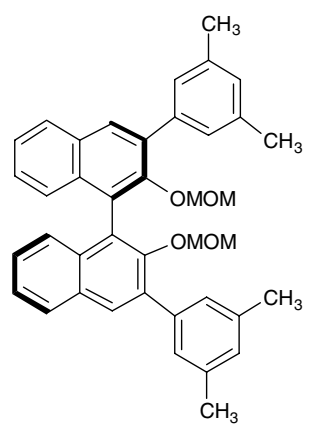

Following General Procedure B, ( \pm )-3,3'-Dibromo-2,2'-Bis(methoxymethoxy-1,1'binaphthyl (3a) (2.0 g, $3.76 \mathrm{mmol})$ was treated with $\mathrm{Pd}\left(\mathrm{PPh}_{3}\right)_{4}$ (0.43 g, $\left.0.372 \mathrm{mmol}\right)$, 3,5-dimethylphenylboronic acid (2.0 g, $13.34 \mathrm{mmol})$ and $2 \mathrm{M}$ aqueous $\mathrm{Na}_{2} \mathrm{CO}_{3}$ solution (10 $\left.\mathrm{mL}\right)$ in DME (40 mL) in a $100 \mathrm{~mL}$ round bottom flask. Purification was carried by column chromatography $\left(\mathrm{CH}_{2} \mathrm{Cl}_{2}\right.$ /hexane: 1/1) ) and crystallization from $\mathrm{CH}_{2} \mathrm{Cl}_{2} /$ hexane to give colorless crystals (3f) $(2.25$ g) in $93 \%$ yield. $\mathrm{mp} 84-85{ }^{\circ} \mathrm{C}$; IR (KBr): v (max) $1600,1389,1233,1157 \mathrm{~cm}^{-1} ;{ }^{1} \mathrm{H} \mathrm{NMR}\left(\mathrm{CDCl}_{3}\right): \delta$ 7.80-7.97 (ArH, 4 H, m), 7.18-7.48 (ArH, 10 H, m), 7.02-7.08 (ArH, $2 \mathrm{H}, \mathrm{m}), 4.69,4.44\left(\mathrm{OCH}_{2} \mathrm{O}, 4\right.$ $\left.\mathrm{H}, \mathrm{d}, \mathrm{AB}, \mathrm{J}_{\mathrm{AB}}=5.8 \mathrm{~Hz}\right), 2.40\left(\mathrm{ArCH}_{3}, 12 \mathrm{H}, \mathrm{s}\right), 2.36\left(\mathrm{OCH}_{3}, 6 \mathrm{H}, \mathrm{s}\right) ;{ }^{13} \mathrm{C} \mathrm{NMR}\left(\mathrm{CDCl}_{3}\right): \delta 151.4$, $139.0,137.7,135.7,133.6,130.8,130.5,128.9,127.7,127.4,126.4,126.1,125.0,98.5,55.8,21.4$; MS m/e (relative intensity): $585\left(\mathrm{M}^{+}+3,1\right), 584\left(\mathrm{M}^{+}+2,4\right), 583\left(\mathrm{M}^{+}+1,15\right), 582\left(\mathrm{M}^{+}, 35\right), 518(12)$, 507 (41), 506 (100), 478 (28); Anal. Calcd for $\mathrm{C}_{40} \mathrm{H}_{38} \mathrm{O}_{4}$ : C, 82.44; H, 6.57. Found: C, 82.55; H, 6.50. (R)-3,3'-Dibromo-2,2'-bis(methoxymethoxy)-1,1'-binaphthyl was used to afford $(R)-3 f . m p$ $81-83{ }^{\circ} \mathrm{C}$. The sample showed $[\alpha]^{25}{ }_{578}-36.8(\mathrm{c}=1.1, \mathrm{THF})$. 
(R)-3,3'-Bis[3,5-di(tert-butyl)phenyl]-2,2'-Bis(methoxymethoxy)-1,1'-binaphthyl (3g).

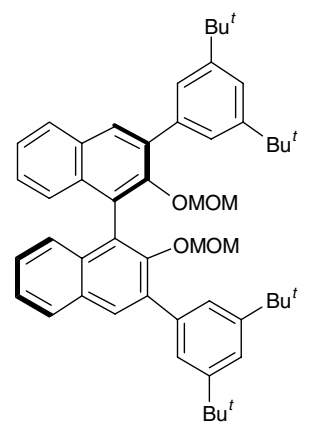

Following General Procedure B, $(R)-3,3$ '-Dibromo-2,2'-Bis(methoxymethoxy-1,1'binaphthyl (3a) $(2.50 \mathrm{~g}, 4.70 \mathrm{mmol})$ was treated with $\mathrm{Pd}\left(\mathrm{PPh}_{3}\right)_{4}(0.54 \mathrm{~g}, 0.470 \mathrm{mmol})$, 3,5-di(tert-butyl)phenylboronic acid (3.85 g, $16.40 \mathrm{mmol})$ and $2 \mathrm{M}$ aqueous $\mathrm{Na}_{2} \mathrm{CO}_{3}$ solution (12.5 $\mathrm{mL})$ in DME $(50 \mathrm{~mL})$ in a $100 \mathrm{~mL}$ round bottom flask. Purification was carried by column chromatography $\left(\mathrm{CH}_{2} \mathrm{Cl}_{2} /\right.$ hexane: $\left.1 / 1\right)$ and crystallization from $\mathrm{CH}_{2} \mathrm{Cl}_{2} /$ hexane to give colorless crystals (3g) (3.5 g) in 99\% yield. mp 97-99 ${ }^{\circ} \mathrm{C} ; \mathrm{IR}(\mathrm{KBr}): v(\max ) 1595,1390,1244,1157 \mathrm{~cm}^{-1} ;{ }^{1} \mathrm{H}$ NMR ( $\left.\mathrm{CDCl}_{3}\right): \delta$ 7.84-8.02 (ArH, $\left.4 \mathrm{H}, \mathrm{m}\right)$, 7.54-7.64 (ArH, $\left.4 \mathrm{H}, \mathrm{m}\right)$, 7.20-7.50 (ArH, $\left.8 \mathrm{H}, \mathrm{m}\right), 4.42$, $4.40\left(\mathrm{OCH}_{2} \mathrm{O}, 4 \mathrm{H}, \mathrm{d}, \mathrm{AB}, \mathrm{J}_{\mathrm{AB}}=5.7 \mathrm{~Hz}\right), 2.37\left(\mathrm{OCH}_{3}, 6 \mathrm{H}, \mathrm{s}\right), 1.39\left(\left(\mathrm{CH}_{3}\right)_{3}, 18 \mathrm{H}, \mathrm{s}\right) ;{ }^{13} \mathrm{C} \mathrm{NMR}$ $\left(\mathrm{CDCl}_{3}\right): \delta 151.6,150.6,138.2,136.4,133.5,130.8,130.2,127.8,126.5,126.4,126.0,124.9,123.9$ 121.0, 98.3, 55.7, 34.9, 31.5; Anal. Calcd for $\mathrm{C}_{52} \mathrm{H}_{62} \mathrm{O}_{4}$ : C, 83.16; H, 8.32. Found: C, 82.96; H, 8.19. The sample showed $[\alpha]^{25} 578-73.8(\mathrm{c}=1.1$, THF $)$.

(R)-3,3'-Bis[3,5-bis(trifluoromethyl)phenyl]-2,2'-Bis(methoxymethoxy)-1,1'-binaphthyl (3h). 


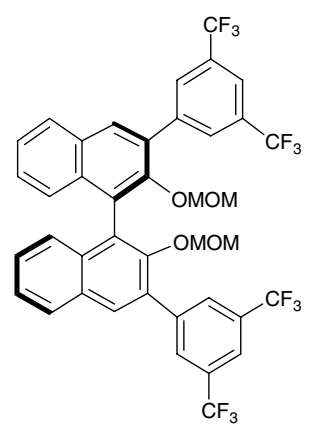

Following General Procedure B, $(R)-3,3$ '-Dibromo-2,2'-Bis(methoxymethoxy-1,1'binaphthyl (3a) $(2.36 \mathrm{~g}, 4.43 \mathrm{mmol})$ was treated with $\mathrm{Pd}\left(\mathrm{PPh}_{3}\right)_{4}(0.512 \mathrm{~g}, 0.443 \mathrm{mmol})$, 3,5-bis(trifluoromethyl)phenylboronic acid $(4.0 \mathrm{~g}, 15.50 \mathrm{mmol})$ and $2 \mathrm{M}$ aqueous $\mathrm{Na}_{2} \mathrm{CO}_{3}$ solution $(11.7 \mathrm{~mL})$ in DME $(50 \mathrm{~mL})$ in a $100 \mathrm{~mL}$ round bottom flask. Purification was carried by column chromatography $\left(\mathrm{CH}_{2} \mathrm{Cl}_{2}\right.$ /hexane: $\left.2 / 3\right)$ and crystallization from $\mathrm{CH}_{2} \mathrm{Cl}_{2} /$ hexane to give slightly yellow crystals $(R)-3 \mathbf{h}(3.0 \mathrm{~g})$ in $85 \%$ yield. mp $69-71{ }^{\circ} \mathrm{C}$; $\mathrm{IR}(\mathrm{KBr}): v(\max ) 1621,1377,1281$, $1153 \mathrm{~cm}^{-1} ;{ }^{1} \mathrm{H}$ NMR $\left(\mathrm{CDCl}_{3}\right): \delta$ 8.20-8.32 (ArH, $\left.4 \mathrm{H}, \mathrm{m}\right), 7.86-8.08(\mathrm{ArH}, 6 \mathrm{H}, \mathrm{m}), 7.20-7.56(\mathrm{ArH}$, $6 \mathrm{H}, \mathrm{m}), 4.43,4.37\left(\mathrm{OCH}_{2} \mathrm{O}, 4 \mathrm{H}, \mathrm{d}, \mathrm{AB}, \mathrm{J}_{\mathrm{AB}}=6.0 \mathrm{~Hz}\right), 2.50\left(\mathrm{OCH}_{3}, 6 \mathrm{H}, \mathrm{s}\right) ;{ }^{13} \mathrm{C} \mathrm{NMR}\left(\mathrm{CDCl}_{3}\right): \delta$ $151.3,141.2,134.2,132.8,132.0,131.5,131.1,130.7,130.0,128.3,127.5,126.4,126.3,125.9$, 125.6, 121.1, 99.2, 56.2; ${ }^{19} \mathrm{~F}$ NMR $\left(\mathrm{CDCl}_{3}\right): \delta$-63.43; Anal. Calcd for $\mathrm{C}_{40} \mathrm{H}_{26} \mathrm{~F}_{12} \mathrm{O}_{4}: \mathrm{C}, 60.16 ; \mathrm{H}$, 3.28. Found: C, $60.35 ; \mathrm{H}, 3.17$. The sample showed $[\alpha]^{25}{ }_{578}-83.6(\mathrm{c}=1.1$, THF).

\section{( \pm )- and (R)-3,3'-Bis(2-naphthyl)-2,2'-Bis(methoxymethoxy)-1,1'-binaphthyl (3i).}

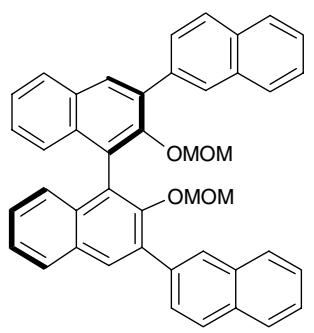

Following General Procedure B, ( \pm )-3,3'-Dibromo-2,2'-Bis(methoxymethoxy-1,1'- 
binaphthyl (3a) (1.2 g, $2.25 \mathrm{mmol})$ was treated with $\mathrm{Pd}\left(\mathrm{PPh}_{3}\right)_{4}$ (0.26 g, $\left.0.225 \mathrm{mmol}\right)$, 2-naphthylboronic acid $(2.02 \mathrm{~g}, 11.76 \mathrm{mmol})$ and $2 \mathrm{M}$ aqueous $\mathrm{Na}_{2} \mathrm{CO}_{3}$ solution $(5.9 \mathrm{~mL})$ in DME $(25 \mathrm{~mL})$ in a $100 \mathrm{~mL}$ round bottom flask. Purification was carried by column chromatography $\left(\mathrm{CH}_{2} \mathrm{Cl}_{2}\right.$ /hexane: 1/1) and crystallization from $\mathrm{CH}_{2} \mathrm{Cl}_{2} /$ hexane to give colorless crystals $(3 \mathbf{i})(1.4 \mathrm{~g})$ in $85 \%$ yield. mp 103-104 ${ }^{\circ} \mathrm{C}$; IR (KBr): v (max) 1597, $1154 \mathrm{~cm}^{-1} ;{ }^{1} \mathrm{H}$ NMR $\left(\mathrm{CDCl}_{3}\right): \delta 8.14-8.28$ (ArH, 2 H, m), 8.01-8.10 (ArH, 2 H, m), 7.70-8.01 (ArH, 10 H, m), 7.22-7.66 (ArH, 10 H, m), 4.45, $4.44\left(\mathrm{OCH}_{2} \mathrm{O}, 4 \mathrm{H}, \mathrm{d}, \mathrm{AB}, \mathrm{J}_{\mathrm{AB}}=5.9 \mathrm{~Hz}\right), 2.32\left(\mathrm{OCH}_{3}, 6 \mathrm{H}, \mathrm{s}\right) ;{ }^{13} \mathrm{C} \mathrm{NMR}\left(\mathrm{CDCl}_{3}\right): \delta 151.5,136.7$, $135.3,133.7,133.5,132.5,130.9,128.1,128.0,127.9,127.7,127.6,126.6,126.5,126.4,126.1$, 126.0, 125.2, 98.6, 55.9; MS m/e (relative intensity): 627 ( $\left.\mathrm{M}^{+}, 1\right), 271$ (22), 270 (100), 269 (30), 268 (15), 239 (22); Anal. Calcd for $\mathrm{C}_{44} \mathrm{H}_{34} \mathrm{O}_{4}$ : C, 84.32; H, 5.47. Found: C, 84.23; H, 5.38. (R)-3,3'-Dibromo-2,2'-bis(methoxymethoxy)-1,1'-binaphthyl was used to afford $(R)-3 \mathbf{i}$ as a yellowish syrup. The sample showed $[\alpha]^{25}{ }_{578}-110(\mathrm{c}=1.1, \mathrm{THF})$.

\section{Preparation of (R)-3,3'-Bis(trifluoromethyl)-2,2' -Bis(methoxymethoxy)-1,1'-Binaphthyls (3j).}

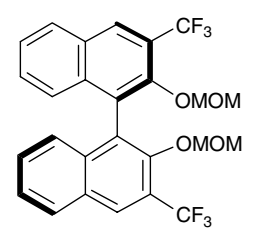

A mixture of $\mathrm{FSO}_{2} \mathrm{CF}_{2} \mathrm{CO}_{2} \mathrm{Me}(1.63 \mathrm{~mL}, 12.78 \mathrm{mmol}), \mathrm{CuI}(1.46 \mathrm{~g}, 7.67 \mathrm{mmol}), \mathrm{HMPA}$ (2.22 mL, $12.78 \mathrm{mmol}$ ) and (R)-3,3'-diiodo-2,2'-dis(methoxymethoxy-1,1'-binaphthyl (yy3) (2.0 g, $3.19 \mathrm{mmol})$ in $\mathrm{DMF}(40 \mathrm{~mL})$ was stirred under argon atmosphere for $6 \mathrm{~h}$ at $70{ }^{\circ} \mathrm{C}$. The reaction mixture was then cooled to room temperature. It was diluted with $\mathrm{CH}_{2} \mathrm{Cl}_{2}(400 \mathrm{~mL})$, the solution washed with water $(3 \times 200 \mathrm{~mL})$, dried over $\mathrm{Na}_{2} \mathrm{SO}_{4}$, and concentrated to afford a syrup. The purification was done by column chromatography (EtOAc/hexane: 1/20) to give $1.51 \mathrm{~g}$ of pure 
product 3j (93\%). mp $43-45{ }^{\circ} \mathrm{C}$; IR ( $\left.\mathrm{KBr}\right): v(\max ) 1230,1165,1035 \mathrm{~cm}^{-1} ;{ }^{1} \mathrm{H} \mathrm{NMR}\left(\mathrm{CDCl}_{3}\right): \delta$ 8.22-8.32 (ArH, $2 \mathrm{H}, \mathrm{m})$, 7.85-7.98 (ArH, $2 \mathrm{H}, \mathrm{m})$, 7.25-7.55 (ArH, $4 \mathrm{H}, \mathrm{m})$, 7.08-7.25 (ArH, $2 \mathrm{H}$, m), 4.68, $4.44\left(\mathrm{OCH}_{2} \mathrm{O}, 4 \mathrm{H}, \mathrm{d}, \mathrm{AB}, \mathrm{J}_{\mathrm{AB}}=5.5 \mathrm{~Hz}\right), 2.63\left(\mathrm{OCH}_{3}, 6 \mathrm{H}, \mathrm{s}\right) ;{ }^{13} \mathrm{C} \mathrm{NMR}\left(\mathrm{CDCl}_{3}\right): \delta 150.8$, 140.3, 135.6, 129.2, 129.1, 129.0, 128.9, 126.9, 126.2, 126.0, 123.9 (q, J = 30.4 Hz), 123.6 (q, 272.7 $\mathrm{Hz}), 99.7,56.2 ;{ }^{19} \mathrm{~F}$ NMR $\left(\mathrm{CDCl}_{3}\right): \delta$-61.7; Anal. Calcd for $\mathrm{C}_{26} \mathrm{H}_{20} \mathrm{~F}_{6} \mathrm{O}_{4}: \mathrm{C}, 61.18 ; \mathrm{H}, 3.95, \mathrm{~F}, 22.33$. Found: C, 61.10; H, 3.76; F, 22.43. The sample showed $[\alpha]^{25}{ }_{589}-57.0(\mathrm{c}=1.0, \mathrm{THF})$.

\section{General Procedure C: Preparation of 3,3'-Disubstituted-2,2'-Dihydroxy-1,1'-Binaphthyls $\mathbf{4 a - 4 j}$.}

A mixture of 3,3'-disubstituted-2,2'-bis(methoxymethoxy)-1,1'-binaphthyl $(\mathbf{3}, 2.0 \mathrm{mmol})$ and Amberlyst 15 resin $(1.0 \mathrm{~g})$ in $\mathrm{THF} / \mathrm{MeOH}(1: 1)$ was stirred and heated to reflux under argon atomosphere for $15 \mathrm{~h}$, then cooled to room temperature. The resin was removed by filtration and the filtrate was concentrated by rotary evaporation. Subsequent purification gave the product.

\section{(R)-3,3'-Dibromo-2,2'-Dihydroxy-1,1'-Binaphthyl (4a).}

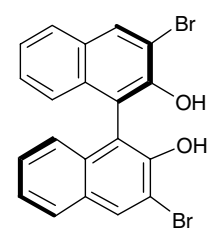

(R)-3,3'-Dibromo-2,2'-bis(methoxymethoxy)-1,1'-binaphthyl (3a) $(2.0 \mathrm{~g}, 3.76 \mathrm{mmol})$ was treated with Amberlyst 15 (2.0 g) in THF/MeOH (100 mL, 1:1) as described in General Procedure C. Purification was carried out by crystallization $\left(\mathrm{CH}_{2} \mathrm{Cl}_{2} /\right.$ hexane) to afford $1.6 \mathrm{~g}$ of a white crystalline product $(R)-\mathbf{4 a}(96 \%)$. mp 256-257 ${ }^{\circ} \mathrm{C}\left(\right.$ lit. $\left.^{6} \mathrm{mp} 243-244{ }^{\circ} \mathrm{C}\right)$; IR $(\mathrm{KBr}): v(\max ) 3479$, $1616,1577,1499,1448,1421,1382,1359,1255,1195,1138 \mathrm{~cm}^{-1} ;{ }^{1} \mathrm{H}$ NMR $\left(\mathrm{CDCl}_{3}\right): \delta 8.20-8.35$ 
(ArH, $2 \mathrm{H}, \mathrm{m})$, 7.74-7.88 (ArH, $2 \mathrm{H}, \mathrm{m})$, 7.05-7.50 (ArH, $6 \mathrm{H}, \mathrm{m}), 5.53(\mathrm{OH}, 2 \mathrm{H}, \mathrm{s}) ;{ }^{13} \mathrm{C} \mathrm{NMR}$ $\left(\mathrm{CDCl}_{3}\right): \delta 148.1,132.8,129.8,127.6,127.4,124.9,124.6,114.7,112.3$; Anal. Calcd for $\mathrm{C}_{20} \mathrm{H}_{12} \mathrm{Br}_{2} \mathrm{O}_{2}$ : C, 54.09; $\mathrm{H}, 2.72$. Found: $\mathrm{C}, 54.05 ; \mathrm{H}, 2.68$. The sample showed $[\alpha]^{25}{ }_{589}+104.7(\mathrm{c}=$ 1.0, THF); $\operatorname{lit}^{6}[\alpha]^{25}+43\left(\mathrm{c}=0.22, \mathrm{CHCl}_{3}\right)$

(R)-3,3'-Diiodo-2,2'-Dihydroxy-1,1'-Binaphthyl (4b).

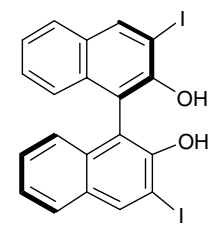

(R)-3,3'-Diiodo-2,2'-bis(methoxymethoxy)-1,1'-binaphthyl (3b) (3.7 g, $5.91 \mathrm{mmol})$ was treated with Amberlyst $15(3.0 \mathrm{~g})$ in THF/MeOH $(160 \mathrm{~mL}, 1: 1)$ as described in General Procedure C. Purification was carried out by column chromatography (EtOAc/hexane: 1:10), and by crystallization (EtOAc/hexane) to afford $3.0 \mathrm{~g}$ of a light yellow crystalline product $(R)-\mathbf{4 b}(94 \%)$. mp 312-314 ${ }^{\circ} \mathrm{C} ; \mathrm{IR}(\mathrm{KBr}): v(\max ) 3483,1565,1355,1177,1141 \mathrm{~cm}^{-1} ;{ }^{1} \mathrm{H}$ NMR $\left(\mathrm{CDCl}_{3}\right): \delta$ 8.40-8.60 (ArH, $2 \mathrm{H}, \mathrm{m}), 7.70-7.90$ (ArH, $2 \mathrm{H}, \mathrm{m}), 7.02-7.50$ (ArH, $6 \mathrm{H}, \mathrm{m}), 5.41(\mathrm{OH}, 2 \mathrm{H}, \mathrm{s}) ;{ }^{13} \mathrm{C}$ NMR $\left(\mathrm{CDCl}_{3}\right): \delta$ 152.0, 140.4, 133.4, 130.7, 128.0, 127.3, 126.8, 125.2, 124.8, 124.4; Anal. Calcd for $\mathrm{C}_{20} \mathrm{H}_{12} \mathrm{I}_{2} \mathrm{O}_{2}$ : C, 44.64; $\mathrm{H}, 2.25$. Found: $\mathrm{C}, 44.58 ; \mathrm{H}, 2.27$. The sample showed $[\alpha]^{25}{ }_{589}+102.7(\mathrm{c}$ $=1.0, \mathrm{THF})$.

(R)-3,3'-Dimethyl-2,2'-Dihydroxy-1,1'-Binaphthyls (4c).

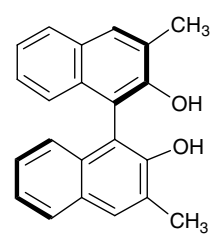

(R)-3,3'-Dimethyl-2,2'-bis(methoxymethoxy)-1,1'-binaphthyl (3c) (1.80 g, $4.47 \mathrm{mmol})$ was 
treated with Amberlyst $15(2.5 \mathrm{~g})$ in $\mathrm{THF} / \mathrm{MeOH}(80 \mathrm{~mL}, 1: 1)$ as described in General Procedure C.

Purification was carried out by column chromatography (EtOAc/hexane: 1:10), and by crystallization $\left(\mathrm{CH}_{2} \mathrm{Cl}_{2} /\right.$ hexane) to afford $1.40 \mathrm{~g}$ of a slightly yellow crystalline product $(R)-4 \mathrm{c}$ (100\%). mp 201-202 ${ }^{\circ} \mathrm{C}$ [ $\left[\mathrm{lit}^{7}\right.$ for $\left.(S)-4 \mathbf{c}, \mathrm{mp} 200-202\right]$; IR, ${ }^{1} \mathrm{H}$ NMR, and ${ }^{13} \mathrm{C}$ NMR were identical with that of $( \pm)-\mathbf{4 c}{ }^{7}$ Anal. Calcd for $\mathrm{C}_{22} \mathrm{H}_{18} \mathrm{O}_{2}$ : C, 84.05; H, 5.77. Found: C, 84.01; H, 5.78. The sample showed $[\alpha]^{25}{ }_{589}+43.4(\mathrm{c}=1.0, \mathrm{THF})$.

(士)- and (R)-3,3'-Diphenyl-2,2'-Dihydroxy-1,1'-Binaphthyl (4d).

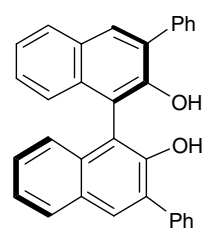

(士)-3,3'-Diphenyl-2,2'-bis(methoxymethoxy)-1,1'-binaphthyl (3d) (6.4 g, 12.15 mmol) was treated with Amberlyst $15(6.0 \mathrm{~g})$ in THF/MeOH $(200 \mathrm{~mL}, 1: 1))$ as described in General Procedure C. Purification was carried out by column chromatography (EtOAc/hexane: 1:10), followed by crystallization $\left(\mathrm{CH}_{2} \mathrm{Cl}_{2}\right.$ /hexane) to afford $4.70 \mathrm{~g}$ of a white crystalline product $( \pm)-\mathbf{4 d}(89 \%)$. mp 209-210 ${ }^{\circ} \mathrm{C}\left[\operatorname{lit}^{7} \mathrm{mp} 210-211{ }^{\circ} \mathrm{C}\right]$; IR, ${ }^{1} \mathrm{H}$ NMR, ${ }^{13} \mathrm{C}$ NMR were identical to that of $( \pm)-(\mathbf{4 d})$ in the literature ${ }^{8}$; Anal. Calcd for $\mathrm{C}_{32} \mathrm{H}_{22} \mathrm{O}_{2}: \mathrm{C}, 87.65 ; \mathrm{H}$, 5.06. Found $\mathrm{C}, 87.55 ; \mathrm{H}, 4.99$. (R)-3,3'-Diphenyl-2,2'-bis(methoxymethoxy)-1,1'-binaphthyl (3d) was used to afford (R)-4d. mp 200-202 ${ }^{\circ} \mathrm{C}\left[\right.$ lit $\left.^{8} \mathrm{mp} 197-198{ }^{\circ} \mathrm{C}\right]$; The sample showed $[\alpha]^{25}{ }_{589}+135.0(\mathrm{c}=1.0, \mathrm{THF})\left[\operatorname{lit}^{8}[\alpha]^{25}{ }_{546}\right.$ $\left.+132.4^{\mathrm{o}}(\mathrm{c}=1.0, \mathrm{THF})\right]$.

( \pm )- and $(R)-3,3$ '-Bis(4-methoxyphenyl)-2,2'-Dihydroxy-1,1'-Binaphthyl (4e). 


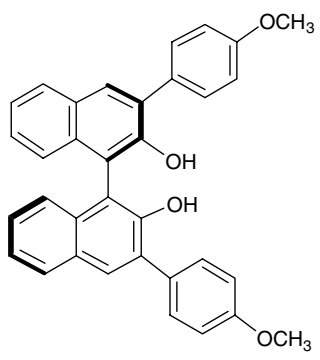

(士)-3,3'-Bis(4-methoxyphenyl)-2,2'-bis(methoxymethoxy)-1,1'-binaphthyl (3e) (1.50 g, $2.56 \mathrm{mmol})$ was treated with Amberlyst $15(1.3 \mathrm{~g})$ in $\mathrm{THF} / \mathrm{MeOH}(80 \mathrm{~mL}, 1: 1)$ as described in General Procedure C. Purification was carried out by crystallization (EtOAc/hexane) to afford 1.15 g of a light yellow crystalline product ( \pm )-4e (90\%). mp 224-225 ${ }^{\circ} \mathrm{C}$; IR (KBr): v (max) 3480, 1606, 1512, 1244, $1177 \mathrm{~cm}^{-1} ;{ }^{1} \mathrm{H} \mathrm{NMR}\left(\mathrm{CDCl}_{3}\right): \delta$ 7.80-8.10 (ArH, $\left.4 \mathrm{H}, \mathrm{m}\right), 7.56-7.75$ (ArH, $\left.4 \mathrm{H}, \mathrm{m}\right)$, 7.12-7.45 (ArH, $6 \mathrm{H}, \mathrm{m}), 6.90-7.06(\mathrm{ArH}, 4 \mathrm{H}, \mathrm{m}), 5.36(\mathrm{OH}, 2 \mathrm{H}, \mathrm{s}), 3.83\left(\mathrm{OCH}_{3}, 6 \mathrm{H}, \mathrm{s}\right) ;{ }^{13} \mathrm{C}$ $\operatorname{NMR}\left(\mathrm{CDCl}_{3}\right): \delta 159.3,150.2,132.7,130.9,130.7,130.3,129.8,129.5,128.3,127.1,124.2,113.9$, 112.4, 55.3; Anal. Calcd for $\mathrm{C}_{34} \mathrm{H}_{26} \mathrm{O}_{4}$ : C, 81.91; H, 5.26. Found: C, 82.02; H, 5.16. (R)-3,3'-Bis(4-methoxyphenyl)-2,2'-bis(methoxymethoxy)-1,1'-binaphthyl was used to afford (R)-4e. mp 109-111 ${ }^{\circ} \mathrm{C}$. The sample showed $[\alpha]^{25}{ }_{578}+50.1(\mathrm{c}=1.0, \mathrm{THF})$.

(土)- and (R)-3,3'-Bis(3,5-dimethylphenyl)-2,2'-Dihydroxy-1,1'-Binaphthyl (4f).

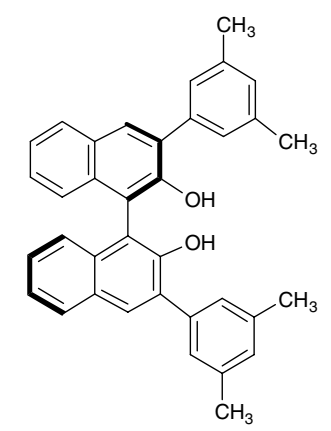

(士)-3,3'-Bis(3,5-dimethylphenyl)-2,2'-bis(methoxymethoxy)-1,1'-binaphthyl (3f) (2.20 g, $3.78 \mathrm{mmol})$ was treated with Amberlyst $15(2.0 \mathrm{~g})$ in $\mathrm{THF} / \mathrm{MeOH}(100 \mathrm{~mL}, 1: 1)$ as described in 
General Procedure C. Purification was carried out by crystallization (THF/hexane) to afford $1.74 \mathrm{~g}$ of a light yellow crystalline product ( \pm )-4f (93\%). mp 299-301 ${ }^{\circ} \mathrm{C}$; IR (KBr): $v(\max ) 3476,1600$, 1497, 1411, 1252, $1211 \mathrm{~cm}^{-1} ;{ }^{1} \mathrm{H}$ NMR $\left(\mathrm{CDCl}_{3}\right): \delta$ 7.82-8.08 (ArH, $\left.4 \mathrm{H}, \mathrm{m}\right), 7.18-7.48(\mathrm{ArH}, 10 \mathrm{H}$, m), 6.98-7.15 (ArH, $2 \mathrm{H}, \mathrm{m}), 5.39(\mathrm{OH}, 2 \mathrm{H}, \mathrm{s}), 2.40\left(\mathrm{ArCH}_{3}, 12 \mathrm{H}, \mathrm{s}\right) ;{ }^{13} \mathrm{C} \mathrm{NMR}\left(\mathrm{CDCl}_{3}\right): \delta$ 150.0, 138.1, 137.3, 133.0, 130.9, 129.5, 129.4, 128.3, 127.3, 127.1, 124.4, 124.1, 112.8, 21.4; Anal. Calcd for $\mathrm{C}_{36} \mathrm{H}_{30} \mathrm{O}_{2}: \quad \mathrm{C}, \quad 87.42 ; \quad \mathrm{H}, \quad$ 6.11. $\quad$ Found: $\quad$ C, $\quad 87.35 ; \quad \mathrm{H}, \quad 5.98$. (R)-3,3'-Bis(3,5-dimethylphenyl)-2,2'-bis(methoxymethoxy)-1,1'-binaphthyl was used to afford $(R)$-4f. mp $124-125{ }^{\circ} \mathrm{C}$. The sample showed $[\alpha]^{25}{ }_{578}+53.2(\mathrm{c}=1.1$, THF $)$.

\section{(R)-3,3'-Bis[3,5-di(tert-butyl)phenyl]-2,2'-Dihydroxy-1,1'-Binaphthyl (4g).}

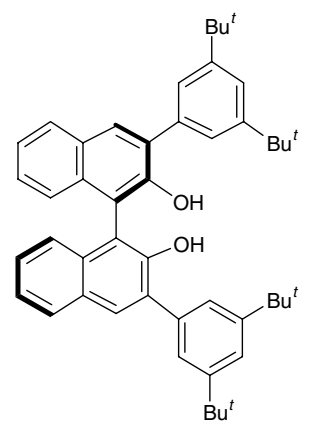

(R)-3,3'-Bis[3,5-di(tert-butyl)phenyl]-2,2'-bis(methoxymethoxy)-1,1'-binaphthyl (3g) (3.0 g, $3.76 \mathrm{mmol})$ was treated with Amberlyst $15(2.0 \mathrm{~g})$ in THF/MeOH $(100 \mathrm{~mL}, 1: 1)$ as described in General Procedure C. Purification was carried out by column chromatography (EtOAc/hexane: 1:10), and by crystallization (EtOAc/hexane) to afford $2.60 \mathrm{~g}$ of a white crystalline product $(R)-\mathbf{4 g}$ (98\%). mp 142-144 ${ }^{\circ} \mathrm{C}$; IR (KBr): v (max) 3490, 1601, 1360, $1209 \mathrm{~cm}^{-1} ;{ }^{1} \mathrm{H}$ NMR $\left(\mathrm{CDCl}_{3}\right): \delta$

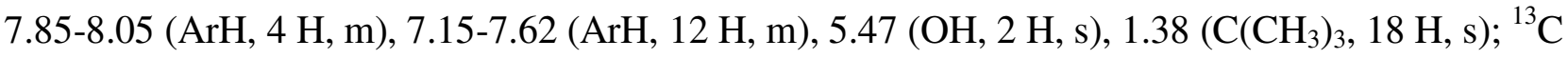
$\operatorname{NMR}\left(\mathrm{CDCl}_{3}\right): \delta 151.0,150.1,136.5,133.0,131.6,130.9,129.4,128.3,127.0,124.5,124.1,123.9$, 121.9, 113.0, 35.0, 31.5; Anal. Calcd for $\mathrm{C}_{48} \mathrm{H}_{54} \mathrm{O}_{2}$ : C, 86.96; H, 8.21. Found: C, 86.86; H, 8.17. 
The sample showed $[\alpha]^{25}{ }_{589}+27.5(\mathrm{c}=1.0, \mathrm{THF})$.

(R)-3,3'-Bis[3,5-bis(trifluoromethyl)phenyl]-2,2'-Dihydroxy-1,1'-Binaphthyl (4h).

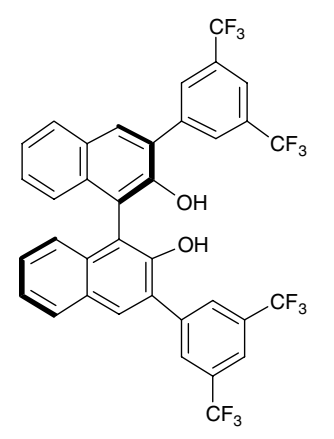

(R)-3,3'-Bis[3,5-bis(trifluoromethyl)phenyl]-2,2'-bis(methoxymethoxy)-1,1'-binaphthyl (3h) (3.0 g, $3.76 \mathrm{mmol})$ was treated with Amberlyst 15 (2.0 g) in THF/MeOH (100 mL, 1:1) as described in General Procedure C. Purification was carried out by column chromatography (EtOAc/hexane: 1:10), and by crystallization (EtOAc/hexane) to afford $2.60 \mathrm{~g}$ of a light yellow crystalline product $(R)-4 h(97 \%) . \operatorname{mp} 97-99{ }^{\circ} \mathrm{C}$; IR (KBr): v (max) 3477, 1603, 1385, 1203, $1165 \mathrm{~cm}^{-1} ;{ }^{1} \mathrm{H}$ NMR $\left(\mathrm{CDCl}_{3}\right): \delta$ 7.80-8.35 (ArH, $\left.10 \mathrm{H}, \mathrm{m}\right), 7.10-7.60(\mathrm{ArH}, 6 \mathrm{H}, \mathrm{m}), 5.37(\mathrm{OH}, 2 \mathrm{H}, \mathrm{s}) ;{ }^{13} \mathrm{C} \mathrm{NMR}$ $\left(\mathrm{CDCl}_{3}\right): \delta 150.0,139.7,133.4,132.4,131.7(\mathrm{q}, \mathrm{J}=33.9 \mathrm{~Hz}), 129.9,129.6,128.9,128.6,127.9(\mathrm{q}, \mathrm{J}$ $=275.5 \mathrm{~Hz}), 125.2,124.1,121.3,112.0 ;{ }^{19} \mathrm{~F} \mathrm{NMR}(\mathrm{CDCl} 3): \delta$-63.49; Anal. Calcd for $\mathrm{C}_{36} \mathrm{H}_{18} \mathrm{O}_{2} \mathrm{~F}_{12}$ : C, 60.86; H, 2.55; F, 32.09. Found: C, 60.80; H, 2.48; F, 31.99. The sample showed $[\alpha]^{25}{ }_{589}-11.9$ (c $=1.1, \mathrm{THF})$. 
(士)- and (R)-3,3'-Bis(2-naphthyl)-2,2'-Dihydroxy-1,1'-Binaphthyl (4i).

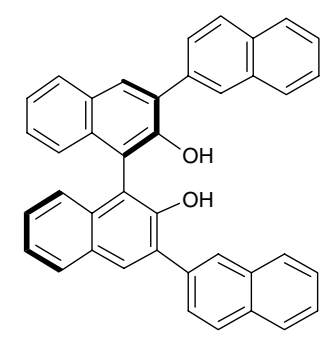

(士)-3,3'-Bis(2-naphthyl)-2,2'-bis(methoxymethoxy)-1,1'-binaphthyl (3i) (1.35 g, $2.15 \mathrm{mmol})$ was treated with Amberlyst $15(1.1 \mathrm{~g})$ in $\mathrm{THF} / \mathrm{MeOH}(80 \mathrm{~mL}, 1: 1)$ as described in General Procedure C. Purification was carried out by column chromatography $\left(\mathrm{CH}_{2} \mathrm{Cl}_{2} /\right.$ hexane: $\left.1: 1\right)$, then by crystallization $\left(\mathrm{CH}_{2} \mathrm{Cl}_{2} /\right.$ hexane) to afford $1.05 \mathrm{~g}$ of a light yellow crystalline product $( \pm)-4 \mathbf{i}$ (91\%). mp 184-185 ${ }^{\circ} \mathrm{C}$; IR (KBr): v (max) 3478, 1615, 1501, 1384, 1226, 1169, $1118 \mathrm{~cm}^{-1} ;{ }^{1} \mathrm{H}$ $\operatorname{NMR}\left(\mathrm{CDCl}_{3}\right): \delta$ 7.70-8.40 (ArH, $\left.14 \mathrm{H}, \mathrm{m}\right), 7.15-7.60(\mathrm{ArH}, 10 \mathrm{H}, \mathrm{m}), 5.46(\mathrm{OH}, 2 \mathrm{H}, \mathrm{s}) ;{ }^{13} \mathrm{C} \mathrm{NMR}$ $\left(\mathrm{CDCl}_{3}\right): \delta 150.3,135.0,133.5,133.0,132.8,131.7,130.6,129.6,128.5,128.2,127.9,127.7,127.4$ 126.3, 124.4, 124.3, 112.5; Anal. Calcd for $\mathrm{C}_{40} \mathrm{H}_{26} \mathrm{O}_{2}$ : C, 89.19; H, 4.86. Found: C, 88.98; H, 5.05. (R)-3,3'-Bis(2-naphthyl)-2,2'-bis(methoxymethoxy)-1,1'-binaphthyl was used to afford $(R)-4 \mathbf{i}: \mathrm{mp}$ $217-218{ }^{\circ} \mathrm{C}$ (dec.). The sample showed $[\alpha]^{25} 578-32.9(\mathrm{c}=1.0, \mathrm{THF})$.

\section{(R)-3,3'-Bis(trifluoromethyl)-2,2'-Dihydroxy-1,1'-Binaphthyl (4j).}

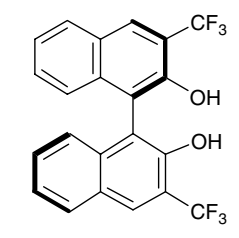

(R)-3,3'-Bis(trifluoromethyl)-2,2'-bis(methoxymethoxy)-1,1'-binaphthyl (3j) (1.60 g, 3.13 mmol) was treated with Amberlyst 15 (1.6 g) in THF/MeOH (80 mL, 1:1) as described in General Procedure C. Purification was carried out by column chromatography (EtOAc/hexane: 1:10), and 
by crystallization $\left(\mathrm{CH}_{2} \mathrm{Cl}_{2} /\right.$ hexane) to afford $1.30 \mathrm{~g}$ of a white crystalline product $(R)-\mathbf{4 j}(98 \%)$. $\mathrm{mp}$ 240-242 ${ }^{\circ} \mathrm{C}$; IR (KBr): v (max) 3490, 1545, 1365, $1179 \mathrm{~cm}^{-1} ;{ }^{1} \mathrm{H}$ NMR $\left(\mathrm{CDCl}_{3}\right): \delta 8.30-8.48(\mathrm{ArH}$, $2 \mathrm{H}, \mathrm{m}), 7.90-8.10(\mathrm{ArH}, 2 \mathrm{H}, \mathrm{m}), 7.00-7.62(\mathrm{ArH}, 6 \mathrm{H}, \mathrm{m}), 5.30(\mathrm{OH}, 2 \mathrm{H}, \mathrm{s}) ;{ }^{13} \mathrm{C} \mathrm{NMR}\left(\mathrm{CDCl}_{3}\right): \delta$ 149.4, 134.6, 130.4 (q, J = 5.5 Hz), 130.0, 129.7, 127.9, 125.5, 123.9, 123.3 (q, J = 272.2 Hz), 118.9 $(\mathrm{q}, \mathrm{J}=30.9 \mathrm{~Hz}), 112.2 ;{ }^{19} \mathrm{~F}$ NMR $\left(\mathrm{CDCl}_{3}\right): \delta$-63.38; Anal. Calcd for $\mathrm{C}_{22} \mathrm{H}_{12} \mathrm{~F}_{6} \mathrm{O}_{2}: \mathrm{C}, 62.57 ; \mathrm{H}, 2.86$; F, 26.99. Found: C, 62.50; H, 2.82; F, 26.88. MS m/e (relative intensity): $423\left(\mathrm{M}^{+}+1,16\right), 422\left(\mathrm{M}^{+}\right.$, 78), 403 (13), 385 (17), 353 (17), 335 (14), 307 (14), 305 (18), 257 (17), 253 (13), 208 (23), 207 (100), 202 (15), 192 (18), 191 (29), 183 (17), 177 (20), 176 (14), 168 (42), 167 (35), 166 (22), 164 (13), 162 (20), 153 (46), 152 (35), 144 (25), 142 (18), 140 (14), 139 (14), 138 (37), 133 (19), 128 (18), 127 (23), 125 (13), 122 (13), 119 (21), 118 (15), 113 (22), 112 (13), 96 (27), 77 (14), 75 (19), 73 (23), 69 (20). The sample showed $[\alpha]^{25}{ }_{589}+72.8(\mathrm{c}=1.0$, THF).

\section{General Procedure D: Allylboration of Aldehydes and Ketones using Binaphthol-modified}

Allylboronates. To a solution of triallylborane $(0.75 \mathrm{mmol}, 1.5 \mathrm{eq})$ in dry THF $(4 \mathrm{~mL})$ under argon was added the 3,3'-disubstituted BINOL 4 ( $0.9 \mathrm{mmol}, 1.8 \mathrm{eq})$. This mixture was stirred at room temperature for $2 \mathrm{~h}$ and then was heated at reflux for $1 \mathrm{~h}$. The solution was cooled to $-78{ }^{\circ} \mathrm{C}$ (in ketone cases, THF was evaporated, $5 \mathrm{~mL}$ toluene was added). The aldehyde (or ketone) ( 0.5 mmol) was dissolved in $1 \mathrm{~mL}$ of dry THF (toluene for ketones) and added dropwise to the solution. Upon completion of the addition, the solution was stirred at $-78^{\circ} \mathrm{C}$ for $1 \mathrm{~h}\left(-78^{\circ} \mathrm{C} \sim-40{ }^{\circ} \mathrm{C}\right.$ for $48 \mathrm{~h}$ for ketones). The reaction was quenched cold with $1 \mathrm{M} \mathrm{NaOH}$ and allowed to warm to room temperature. The mixture was diluted with $\mathrm{CH}_{2} \mathrm{Cl}_{2}(20 \mathrm{~mL})$ and the organic layer washed with 0.1 $\mathrm{M} \mathrm{NaOH}$ then brine, dried with sodium sulfate. The ligand was recovered by neutralizing the aqueous layer with $1 \mathrm{M} \mathrm{HCl}$ and extracting with $\mathrm{Et}_{2} \mathrm{O}$. The organic layer was evaporated under 
reduced pressure to afford a clear colorless oil, which was purified via flash column chromatography (10:1 hexane:ethyl acetate) to provide the expected homoallylic alcohol in the yields reported in Tables 1, 2, and 3 of the paper. The enantiomeric excesses of most alcohols were determined by HPLC ( $4.6 \times 250 \mathrm{~mm}$ ChiralCel OD) using racemic mixtures prepared with triallylborane as standards.

While excess binaphthol was typically used to ensure that no free triallylborane remained, control experiments using 1.0:1.0 binaphthol:triallylborane gave essentially identical enantioselectivities.

(R)-1-Phenyl-3-buten-1-ol (6a).

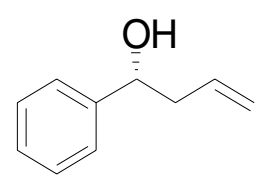

$[\alpha]_{\mathrm{D}}=+43.6$ (c 1.24, benzene, $86 \%$ ee), lit. ${ }^{9}$ for $S$ enantiomer: $[\alpha]_{\mathrm{D}}=-44.92$ (c 7.38, benzene, 96 \%ee); ${ }^{1} \mathrm{H}$ NMR (300 MHz, $\left.\mathrm{CDCl}_{3}\right) \delta 2.06(1 \mathrm{H}, \mathrm{br}), 2.34-2.57(2 \mathrm{H}, \mathrm{m}), 4.70(1 \mathrm{H}, \mathrm{dd}, \mathrm{J}=11.6$, 7.0Hz), 5.11-5.17 $(2 \mathrm{H}, \mathrm{m}), 5.72-5.86(1 \mathrm{H}, \mathrm{m}), 7.26-7.60(5 \mathrm{H}, \mathrm{m})$; \%ee was determined by HPLC (hexane/ $\mathrm{PrOH}=98 / 2$, flow rate $=1 \mathrm{~mL} / \mathrm{min}), \mathrm{t}_{\mathrm{R}}=15.87 \mathrm{~min}(R), \mathrm{t}_{\mathrm{R}}=18.90 \mathrm{~min}(S)$.

\section{(R)-l-(4-Methoxyphenyl)-3-buten-l-o1 (6b).}

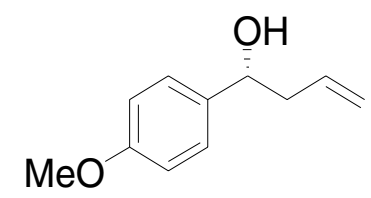

$[\alpha]_{\mathrm{D}}=+62.4\left(\mathrm{c} 0.75, \mathrm{CHCl}_{3}, 93 \%\right.$ ee), lit. ${ }^{10}:[\alpha]_{\mathrm{D}}=+30.5$ (c 1.0, benzene, $95 \%$ ee); ${ }^{1} \mathrm{H}$ NMR $(300$ $\left.\mathrm{MHz}, \mathrm{CDCl}_{3}\right) \delta 2.06(1 \mathrm{H}, \mathrm{br} \mathrm{s}), 2.44(2 \mathrm{H}, \mathrm{t}, \mathrm{J}=6.6 \mathrm{~Hz}), 3.74(3 \mathrm{H}, \mathrm{s}), 4.59(1 \mathrm{H}, \mathrm{dd}, \mathrm{J}=6.3,6.6 \mathrm{~Hz})$, 5.08-5.13 (2 H, m), 5.53-5.93 (1 H, m), $6.82(2 \mathrm{H}, \mathrm{d}, \mathrm{J}=8.7 \mathrm{~Hz}), 2.66-2.62(1 \mathrm{H}, \mathrm{br} \mathrm{s}) ; 7.20(2 \mathrm{H}, \mathrm{d}$, $\mathrm{J}=8.7 \mathrm{~Hz}$ ). \%ee was determined by HPLC (hexane $/ \mathrm{PrOH}=98 / 2$, flow rate $=1 \mathrm{~mL} / \mathrm{min}$ ), $\mathrm{t}_{\mathrm{R}}=$ $21.09 \min (R), \mathrm{t}_{\mathrm{R}}=26.12 \min (S)$. 
(R)-1-(4-Chlorophenyl)-3-buten-1-ol (6c).

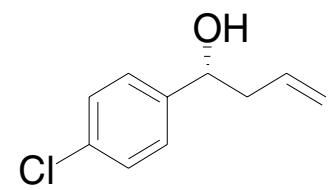

$[\alpha]_{\mathrm{D}}=+61.4\left(\mathrm{c} 1.17, \mathrm{CHCl}_{3}, 94 \% \mathrm{ee}\right), \mathrm{lit}^{11}:[\alpha]_{\mathrm{D}}=+26.4\left(\mathrm{c} 0.38, \mathrm{C}_{6} \mathrm{H}_{6}, 98 \%\right.$ ee); ${ }^{1} \mathrm{H} \mathrm{NMR}(300$ $\left.\mathrm{MHz}_{\mathrm{CDCl}}\right)^{2} 82.02(1 \mathrm{H}, \mathrm{br} \mathrm{s}), 2.37-2.53(2 \mathrm{H}, \mathrm{m}), 4.68(1 \mathrm{H}, \mathrm{dd}, \mathrm{J}=7.5,5.2 \mathrm{~Hz}), 5.12-5.17(2 \mathrm{H}, \mathrm{m})$, 5.68-5.83 (1 H, m), 7.19-7.31 (4H, m); \%ee was determined by HPLC (hexane/iPrOH = 98/2, flow rate $=0.6 \mathrm{~mL} / \mathrm{min}), \mathrm{t}_{\mathrm{R}}=23.19 \mathrm{~min}(S), \mathrm{t}_{\mathrm{R}}=25.29 \mathrm{~min}(R)$.

(R)-1-(4-Nitrophenyl)-3-buten-1-ol (6d).

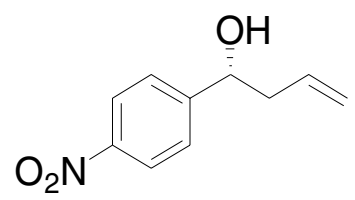

$[\alpha]_{\mathrm{D}}=+58.6\left(\mathrm{c} 0.5, \mathrm{CHCl}_{3}, 91 \%\right.$ ee $), \mathrm{lit}^{12}:[\alpha]_{\mathrm{D}}=+40.1\left(\mathrm{c} 1.4, \mathrm{CHCl}_{3}, 89 \% \mathrm{ee}\right) ;{ }^{1} \mathrm{H} \mathrm{NMR}(300 \mathrm{MHz}$, $\left.\mathrm{CDCl}_{3}\right) 82.22(1 \mathrm{H}$, br s), 2.38-2.60 (2H, m), $4.85(1 \mathrm{H}, \mathrm{dd}, \mathrm{J}=4.8,7.6 \mathrm{~Hz}), 5.14-5.20(2 \mathrm{H}, \mathrm{m})$, 5.70-5.84 (1H, m), $7.52(2 \mathrm{H}, \mathrm{d}, 8.4 \mathrm{~Hz}), 8.05(2 \mathrm{H}, \mathrm{d}, 8.4 \mathrm{~Hz})$; \%ee was determined by ${ }^{19} \mathrm{~F}$ NMR of its corresponding $R$-Mosher esters (282 $\mathrm{MHz}, \mathrm{CDCl}_{3}$ ), 8-70.83 (for $R R$ diastereomer), -70.96 (for $S R$ diastereomer).

(R)-1-(4-Trifluoromethylphenyl)-3-buten-1-ol (6e).

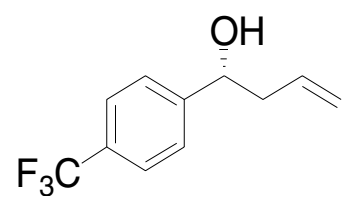

$[\alpha]_{\mathrm{D}}=+53.2\left(\mathrm{c} 0.82, \mathrm{CHCl}_{3}, 93 \%\right.$ ee $), \mathrm{lit}^{12}:[\alpha]_{\mathrm{D}}=+43.1\left(\mathrm{c} 1.24, \mathrm{CHCl}_{3}, 96 \%\right.$ ee $) ;{ }^{1} \mathrm{H}$ NMR $(300$ $\left.\mathrm{MHz}, \mathrm{CDCl}_{3}\right) 82.37(1 \mathrm{H}$, br s), 2.40-2.53 (2H, m), 4.74-4.76(1H, m), 5.13-5.17(2H, m), 5.70-5.83(1H, m), $7.44(2 \mathrm{H}, \mathrm{d}, 8.1 \mathrm{~Hz}), 7.58(2 \mathrm{H}, \mathrm{d}, 8.1 \mathrm{~Hz})$; \%ee was determined by ${ }^{19} \mathrm{~F} \mathrm{NMR}$ of its 
corresponding $R$-Mosher esters (282 MHz, $\mathrm{CDCl}_{3}$ ), 8-70.89 (for $R R$ diastereomer), -71.06 (for $S R$ diastereomer).

(R)-1-Phenyl-1,5-hexadien-3-ol (6f).

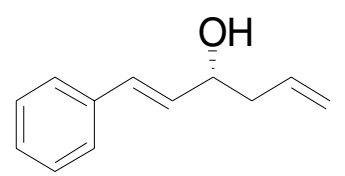

$[\alpha]_{\mathrm{D}}=-6.3\left(\mathrm{c} 0.8, \mathrm{Et}_{2} \mathrm{O}, 75 \% \mathrm{ee}\right), \mathrm{lit}^{10}:[\alpha]_{\mathrm{D}}=-12.3\left(\mathrm{c} 1.0, \mathrm{Et}_{2} \mathrm{O}, 87 \% \mathrm{ee}\right) ;{ }^{1} \mathrm{H}$ NMR $(300 \mathrm{MHz}$, $\left.\mathrm{CDCl}_{3}\right) 81.90(1 \mathrm{H}, \mathrm{br} \mathrm{s}), 2.32-2.49(2 \mathrm{H}, \mathrm{m}), 4.34-4.38(1 \mathrm{H}, \mathrm{m}), 5.15-5.21(2 \mathrm{H}, \mathrm{m}), 5.81-5.87(1 \mathrm{H}$, m), $6.25(1 \mathrm{H}, \mathrm{dd}, \mathrm{J}=6.0,16.0 \mathrm{~Hz}), 6.60(1 \mathrm{H}, 16.0 \mathrm{~Hz}), 7.21-7.40(5 \mathrm{H}, \mathrm{m}) ; \%$ ee was determined by

${ }^{19} \mathrm{~F}$ NMR of its corresponding $R$-Mosher ester [(S)-MTPA-Cl, Et ${ }_{3} \mathrm{~N}$, cat. DMAP, $\left.282 \mathrm{MHz}, \mathrm{CDCl}_{3}\right]$, 8-71.04 (for $R R$ diastereomer), -71.15 (for $S R$ diastereomer).

(R)-1-Cyclohexyl-3-buten-1-ol (6g).

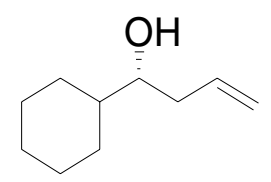

$[\alpha]_{\mathrm{D}}=+1.1\left(\mathrm{c} 0.53, \mathrm{CHCl}_{3}, 75 \% \mathrm{ee}\right), \mathrm{lit}^{10}:[\alpha]_{\mathrm{D}}=+13.7$ (c 1.0, Ethanol, $93 \%$ ee $) ;{ }^{1} \mathrm{H}$ NMR $(300$ $\left.\mathrm{MHz}, \mathrm{CDCl}_{3}\right)$ 80.95-1.85 (11H, m), 2.06-2.27(3H, m), 3.33-3.39(1H, m), 5.08-5.13 (2H, m), 5.74-5.88 $(1 \mathrm{H}, \mathrm{m})$; \%ee was determined by HPLC analysis (hexane/iPrOH $=99.9 / 0.1$, flow rate $=1$ $\mathrm{mL} / \mathrm{min})$ of its corresponding benzoate ester, $\mathrm{t}_{\mathrm{R}}=7.62 \min (R), \mathrm{t}_{\mathrm{R}}=8.65 \mathrm{~min}(S)$.

(R)-2-Phenyl-4-penten-2-ol (7a).

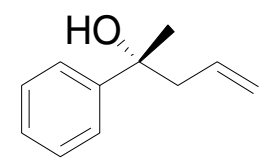

$[\alpha]_{\mathrm{D}}=+61.7\left(\mathrm{c} 0.36, \mathrm{CHCl}_{3}, 91 \%\right.$ ee $), \mathrm{lit}^{13}:[\alpha]_{\mathrm{D}}=+36.3\left(\mathrm{c} 0.843, \mathrm{CHCl}_{3}, 61 \% \mathrm{ee}\right) ;{ }^{1} \mathrm{H}$ NMR $(300$ 
$\left.\mathrm{MHz}, \mathrm{CDCl}_{3}\right) 81.54(3 \mathrm{H}, \mathrm{s}), 1.99(1 \mathrm{H}, \mathrm{br} \mathrm{s}), 2.49(1 \mathrm{H}, \mathrm{dd}, \mathrm{J}=8.4,13.7 \mathrm{~Hz}), 2.68(1 \mathrm{H}, \mathrm{dd}, \mathrm{J}=13.7$, $6.9 \mathrm{~Hz}), 5.10-5.15(2 \mathrm{H}, \mathrm{m}), 5.54-5.68(1 \mathrm{H}, \mathrm{m}), 7.20-2.44(5 \mathrm{H}, \mathrm{m})$; \%ee was determined by HPLC (hexane $/ \mathrm{iPrOH}=99 / 1$, flow rate $=1 \mathrm{~mL} / \mathrm{min}), \mathrm{t}_{\mathrm{R}}=12.58 \min (R), \mathrm{t}_{\mathrm{R}}=13.98 \min (S)$.

\section{(R)-1-Phenyl-1-(2-propenyl)-oxirane ${ }^{14}$}

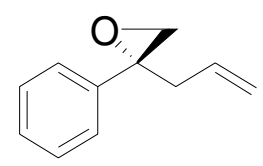

$(R)$-1-Phenyl-1-(2-propenyl)-oxirane was obtained from the allyboration of $\alpha$-bromoacetophenone after workup with $1 \mathrm{M} \mathrm{NaOH} .[\alpha]_{\mathrm{D}}=-29.8\left(\mathrm{c} 0.84, \mathrm{CHCl}_{3}, 93 \%\right.$ ee $) ;{ }^{1} \mathrm{H} \mathrm{NMR}\left(300 \mathrm{MHz}, \mathrm{CDCl}_{3}\right)$ $82.64(1 \mathrm{H}, \mathrm{dd}, \mathrm{J}=7.8,15.0 \mathrm{~Hz}), 2.75(1 \mathrm{H}, \mathrm{d}, \mathrm{J}=5.2 \mathrm{~Hz}), 2.88(1 \mathrm{H}, \mathrm{dd}, \mathrm{J}=6.3,15.0 \mathrm{~Hz}), 2.99(1 \mathrm{H}, \mathrm{d}$, $\mathrm{J}=5.2 \mathrm{~Hz})$, 5.06-5.14 $(2 \mathrm{H}, \mathrm{m}), 5.70-5.84(1 \mathrm{H}, \mathrm{m}), 7.25-7.38(5 \mathrm{H}, \mathrm{m})$; \%ee was determined by HPLC $($ hexane $/ \mathrm{iPrOH}=99.5 / 0.5$, flow rate $=1 \mathrm{~mL} / \mathrm{min}), \mathrm{t}_{\mathrm{R}}=6.54 \mathrm{~min}(R), \mathrm{t}_{\mathrm{R}}=7.93 \mathrm{~min}(S)$. This compound has not been previously reported in enantiomerically enriched form; the absolute configuration has been assigned based on correlation with results for acetophenone and the assumption that $-\mathrm{CH}_{2} \mathrm{Br}\left(\mathrm{A} \text { value }=1.79 \mathrm{kcal} \mathrm{mol}^{-1}\right)^{15}$ and $-\mathrm{CH}_{3}(\mathrm{~A}$ value $=1.74)$ behave similarly in the allylation.

(R)-2-(4'-Methoxyphenyl)-4-penten-2-ol (7c). ${ }^{16}$

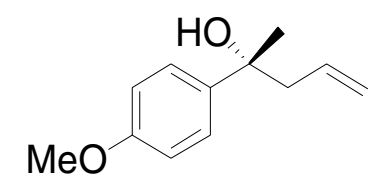

$[\alpha]_{\mathrm{D}}=+65.2\left(\mathrm{c} 0.71, \mathrm{CHCl}_{3}, 98 \% \mathrm{ee}\right) ;{ }^{1} \mathrm{H} \mathrm{NMR}\left(300 \mathrm{MHz}, \mathrm{CDCl}_{3}\right) \delta 1.51(3 \mathrm{H}, \mathrm{s}), 2.02(1 \mathrm{H}, \mathrm{br}, \mathrm{s})$, $2.46(1 \mathrm{H}, \mathrm{dd}, \mathrm{J}=8.1,13.1 \mathrm{~Hz}), 2.64(1 \mathrm{H}, \mathrm{dd}, \mathrm{J}=6.0,13.1 \mathrm{~Hz}), 3,78(3 \mathrm{H}, \mathrm{s}), 5.07-5.13(2 \mathrm{H}, \mathrm{m})$, 5.54-5.68 $(1 \mathrm{H}, \mathrm{m}), 6.85(2 \mathrm{H}, \mathrm{d}, 8.4 \mathrm{~Hz}), 7.40(2 \mathrm{H}, \mathrm{d}, 8.4 \mathrm{~Hz})$; \%ee was determined by HPLC (hexane $/ \mathrm{iPrOH}=99.5 / 0.5$, flow rate $=0.6 \mathrm{~mL} / \mathrm{min}), \mathrm{t}_{\mathrm{R}}=34.01 \mathrm{~min}(R), \mathrm{t}_{\mathrm{R}}=37.98 \min (S)$. 
(R)-2-(4'-Chlorophenyl)-4-penten-2-ol (7d). ${ }^{16}$

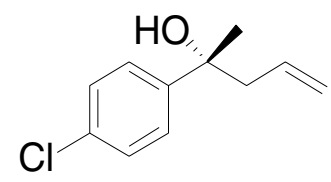

$[\alpha]_{\mathrm{D}}=+63.1\left(\mathrm{c} 0.58, \mathrm{CHCl}_{3},>98 \% \mathrm{ee}\right) ;{ }^{1} \mathrm{H} \mathrm{NMR}\left(300 \mathrm{MHz}, \mathrm{CDCl}_{3}\right) 81.50(3 \mathrm{H}, \mathrm{s}), 2.08(1 \mathrm{H}, \mathrm{br}, \mathrm{s})$, $2.46(1 \mathrm{H}, \mathrm{dd}, \mathrm{J}=6.0,13.2 \mathrm{~Hz}), 2.63(1 \mathrm{H}, \mathrm{dd}, \mathrm{J}=7.8,13.2 \mathrm{~Hz}), 5.09-5.13(2 \mathrm{H}, \mathrm{m}), 5.49-5.65(1 \mathrm{H}$, m), 7.27-7.36 $(4 \mathrm{H}, \mathrm{m})$; \%ee was determined by HPLC (hexane/iPrOH $=99.5 / 0.5$, flow rate $=0.6$ $\mathrm{mL} / \mathrm{min}), \mathrm{t}_{\mathrm{R}}=33.49 \min (S), \mathrm{t}_{\mathrm{R}}=35.03 \mathrm{~min}(R)$. The minor $(S)$ isomer was not detected but its retention time was determined using authentic racemate.

(R)-1-phenyl-3-hydroxy-3-methyl-1,5-hexandiene (7e). ${ }^{16}$

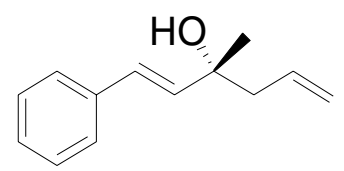

$[\alpha]_{\mathrm{D}}=+65.8\left(\mathrm{c} 1.1, \mathrm{CHCl}_{3}, 75 \%\right.$ ee $) ;{ }^{1} \mathrm{H} \mathrm{NMR}\left(300 \mathrm{MHz}, \mathrm{CDCl}_{3}\right) \delta 1.38(3 \mathrm{H}, \mathrm{s}), 1.78(1 \mathrm{H}, \mathrm{br}, \mathrm{s})$, 2.31-2.47 (2H, m), 5.12-5.18 (2H, m), 5.69-5.90 (1H, m), $6.29(1 \mathrm{H}, \mathrm{d}, \mathrm{J}=15.9), 6.59(1 \mathrm{H}, \mathrm{d}, \mathrm{J}=$ 15.9),7.21-7.38 $(5 \mathrm{H}, \mathrm{m})$; \%ee was determined by HPLC (hexane/iPrOH $=95 / 5$, flow rate $=1.0$ $\mathrm{mL} / \mathrm{min}), \mathrm{t}_{\mathrm{R}}=11.58 \min (R), \mathrm{t}_{\mathrm{R}}=13.78 \min (S)$.

(R)-2,2,3-Trimethyl- 5-hexen-3-ol (7f). ${ }^{17}$

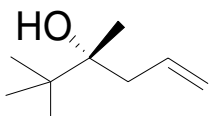

${ }^{1} \mathrm{H}$ NMR (300 MHz, C $\left.6 \mathrm{D}_{6}\right) \mathrm{\delta} 0.97(9 \mathrm{H}, \mathrm{s}), 1.03(3 \mathrm{H}, \mathrm{s}), 1.19(1 \mathrm{H}, \mathrm{br}, \mathrm{s}), 2.06(1 \mathrm{H}, \mathrm{dd}, \mathrm{J}=7.8,13.5$ $\mathrm{Hz}), 2.32(1 \mathrm{H}, \mathrm{dd}, \mathrm{J}=6.9,13.5 \mathrm{~Hz}), 5.02-5.13(2 \mathrm{H}, \mathrm{m}), 5.87-6.00(1 \mathrm{H}, \mathrm{m})$; \%ee was determined by 
${ }^{1} \mathrm{H}$ NMR $\left(300 \mathrm{MHz}, \mathrm{CDCl}_{3}\right)$ in the presence of chiral shift reagent, $\mathrm{Eu}(\mathrm{hfc})_{3}$.

(R)-3-Methyl-1-phenyl-5-hexen-3-ol (7g).

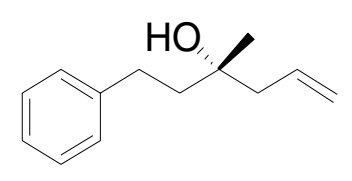

$[\alpha]_{\mathrm{D}}=+6.0\left(\mathrm{c} 1.1, \mathrm{CHCl}_{3}, 51 \%\right.$ ee $)$, literature value for $S$ enantiomer ${ }^{18}:[\alpha]_{\mathrm{D}}=-58.0\left(\mathrm{c} 1.0, \mathrm{CHCl}_{3}\right.$, $92 \%$ ee); ${ }^{1} \mathrm{H}$ NMR (300 MHz, $\left.\mathrm{C}_{6} \mathrm{D}_{6}\right) 80.97(1 \mathrm{H}, \mathrm{s}), 1.07(3 \mathrm{H}, \mathrm{s}), 1.64-1.70(2 \mathrm{H}, \mathrm{m}), 2.12-2.14(2 \mathrm{H}$, $\mathrm{m})$, 2.66-2.70 $(2 \mathrm{H}, \mathrm{m}), 5.02-5.10(2 \mathrm{H}, \mathrm{m}), 5.75-5.90(1 \mathrm{H}, \mathrm{m}), 7.16-7.23(5 \mathrm{H}, \mathrm{m})$; \%ee was determined by HPLC (hexane $/ \mathrm{iPrOH}=95 / 5$, flow rate $=0.6 \mathrm{~mL} / \mathrm{min}), \mathrm{t}_{\mathrm{R}}=16.18 \mathrm{~min}(S), \mathrm{t}_{\mathrm{R}}=$ $17.35 \min (R)$.

\section{References}

1. Chong, J. M.; Shen, L. Synth. Commun. 1998, 28, 2801-2806.

2. Brown, H. C.; Racherla, U. S. J. Org. Chem. 1986, 51, 427-432.

3. Ding, K.; Wang, Y.; Zhang, L.; Wu, Y.; Matsuura, T. Tetrahedron 1996, 52, 1005-1010.

4. (a) Cai, D.; Hughes, D. L.; Verhoeven, T. R.; Reider, P. J. Tetrahedron Lett. 1995, 36, 7991-7994. (b) Cai, D.; Hughe, D. L.; Verhoeven, T. R.; Reider, P. J. Org. Synth.1998, 76, 1-5.

5. Cox, P. J.; Wang, W.; Snieckus, V. Tetrahedron Lett. 1992, 33, 2253-2256.

6. Chow, H.-F.; Wan, C.-W.; Ng, M.-K. J. Org. Chem. 1996, 61, 8712-8714.

7. Cram, D. J.; Helgeson, R. C.; Peacock, S. C.; Kaplan, L. J.; Domeier, L. A.; Moreau, P.; Koga, K.; Mayer, J. M.; Chao, Y.; Siegel, M. G.; Hoffman, D. H.; Sogah, G. D. Y. J. Org. Chem. 1978, 43, 
1930-1946.

8. Lingenfelter, D. S.; Helgeson, R. C.; Cram, D. J. J. Org. Chem. 1981, 46, 393-406.

9. Jadhav, P. K.; Bhat, K. S.; Perumal, P. T.; Brown, H. C. J. Org. Chem. 1986, 51, 432-439.

10. Wadamoto, M.; Ozasa, N.; Yanagisawa, A.; Yamamoto, H. J. Org. Chem. 2003, 68, 5593-5601.

11. Sugimoto, K.; Aoyagi, S.; Kibayashi, C. J. Org. Chem. 1997, 62, 2322-2323.

12. Malkov, A. V.; Dufkova, L.; Farrugia, L.; Kocovsky, P. Angew. Chem. Int. Ed. Engl. 2003, 42, $3674-3677$

13. Yamasaki, S.; Fujii, K.; Wada, R.; Kanai, M.; Shibasaki, M. J. Am. Chem. Soc. 2002, 124, 6536-6537.

14. Pri-Bar, I.; Pearlman, P. S.; Stille, J. K. J. Org. Chem., 1983, 4629-4634

15. Eliel, E. L.; Wilen, S. H. Stereochemistry of Organic Compounds; Wiley: New York, 1994, p. 697.

16. Casolari, S.; D'Addario, D.; Tagliavini, E. Org. Lett. 1999, 1, 1061-1063

17. Zhang, M.; Jia, Y.; Zhou, J.; Wu, S. Heteroatom Chemistry, 1998, 9, 475-478

18. Tietze, L. F.; Schiemann, K.; Wegner, C.; Wulff, C. Eur. J. Chem.1998, 4, 1862-1869. 